\title{
Progression of NETs Correlating with Tumor-Related Diseases
}

\author{
Le-Meng Zhang, Jian-Hua Chen*
}

\begin{abstract}
As an important component of innate immune system, neutrophil has been involved in many other physiological processes, including tumor-related diseases. In 2004, the phenomenon of NETs was reported for the first time. Extracellular decondensed chromatin, released from activated neutrophils, forms a network structure, which is NETs. This review focuses on the function of NETs in tumor cell proliferation, metastasis, and tumor-associated thrombosis; it also explores the application of NETs specific markers in the diagnosis of pre-thrombotic state and tumor associated diseases; it also explores NETs inhibitor for the treatment of tumor-related diseases. In view of the rapid development of NETs, it may provide new therapeutic targets for tumor-associated thrombosis, and even tumor itself.
\end{abstract}

Keywords: Neutrophil extracellular traps - tumor-related diseases - thrombosis - citrullinated histone

Asian Pac J Cancer Prev, 16 (17), 7431-7434

Neutrophil is an important part of innate immune system, with the ability of chemotaxis and phagocytosis. In addition to the resistance of pathogenic microorganisms, neutrophils are also involved in many pathophysiological processes, including thrombosis, autoimmune diseases and tumor related diseases. Plenty of studies have confirmed that the neutrophils play a pivotal role in tumor cells proliferation, metastasis and the process of thrombosis formation. Either in the animal model or in the human tumor tissues, there are neutrophils infiltrations into the tumor tissues [1-3]. Tumor associated neutrophils can release the elastase, matrix metalloproteinase, cytokine and chemokine, which influence the pathogenesis of malignant diseases at different stages, such as to promote the tumor cell proliferation, angiogenesis and metastasis; cellular toxicity of neutrophils can also induce tumor cell apoptotic cell death (Mantovani, 2009; Gregory and Houghton, 2011; Brandau et al., 2013) .

In addition to phagocytosis, chemotaxis and degranulation, neutrophils can also participate the immune response through extracellular release chromatin, which formed network structure, called neutrophil extracellular traps (NETs). NETs have first been confirmed to kill microorganisms (Brinkmann et al., 2004; Fuchs et al., 2007) . Recently, the role of NETs in tumor related diseases have raised increasing attention. NETs are highly expressed in many kinds of malignant tumor tissues, and are involved in the progression of tumors. Here, we summarize the role of NETs in tumor cell proliferation, distant metastasis, and the mechanism of tumor associated thrombosis. We also explore the application of NETs specific markers in the diagnosis of tumor-associated thrombosis. In view of the rapid development of NETs, it may provide new therapeutic targets for tumor associated thrombosis, and even tumor itself.

\section{Introduction of NETs}

In 2004, Brinkmann et al first reported that, duiring the infection or inflammatory response phase, activated neutrophils can positively extracellular release chromatin and form an extracellular network structure, which is called NETs (Brinkmann et al., 2004; Fuchs et al., 2007) .NETs contain chromatin and granule proteins, including nuclear DNA, histones, matrix metalloproteinase 9 (MMP-9), myeloperoxidase (MPO), neutrophil elastase (NE), cathepsin G (CG). With deoxyribonuclease (DNase) treatment, even without proteolytic enzymes, NETs structure can be decomposed, thus confirming that DNA is the backbone components of NETs (Brinkmann et al., 2004).

The chromatin composition in NETs structure is different from that of inside nucleus. Extracellular chromatin of NETs is depolymerized. Chromatin depolymerization is an essential step in the formation of NETs, and a variety of molecular mechanisms are involved in the regulation of chromatin depolymerization (Brinkmann et al., 2004). It has been demonstrated that NE translocation into the nucleus, which degraded histone and assisted chromatin disaggregation; on the other hand, NE deficient mice fail to form NETs (Papayannopoulos et al., 2010).The product of MPO, hypochlorous acid, is also necessary for the extracellular chromatin release (Palmer et al., 2012); neutrophils without MPO activity cannot generate NETs (Papayannopoulos et al., 2010; Metzler et al., 2011). Wang et al. showed that the process of chromatin depolymerization depends on the histone citrullination, which was regulated by peptide arginine deiminase 4 (PAD4) (Wang et al., 2009). PAD4 catalyzed histone arginine residues citrullination; electrically neutral coralline residues replaced positively charged amino acid

Thoracic Medicine Department 1, Hunan Cancer Hospital, Affiliated to Xiangya Medical School, Central South University, Changsha, Hunan,China*For correspondence: cjh_1000@163.com,497730787@qq.com 
residues, which resulted in staining chromatin structure depolymerization (Li et al., 2010). PAD4 knockout mice are unable to form NETs, and infused with wild-type neutrophils to PAD4 KO mice can form NETs again, thus proving that the release of NETs requires PAD4 (Li et al., 2010). To understand the molecular mechanism of NETs formation will be helpful to provide a potential target for the regulation of NETs level.

NETs are located in the site of infection, through the trap of bacterial, viral particles, parasites, fungi, which prevent the spread of infection. In the NETs structure, the concentrations of antimicrobial molecules, such as histone, antimicrobial peptides and protease, are very high locally, which can effectively degrade the pathogenic factors and kill pathogenic microorganisms (Brinkmann et al., 2004; Fuchs et al., 2007). In addition to elimination of pathogenic microorganisms, NETs also provided unique microenvironment for many pathophysiological situations, including autoimmune disease, thrombotic diseases and tumor-associated diseases (Amulic et al., 2012). NETs provide a unique environment for the destruction of normal immune tolerance, which can cause autoimmune diseases. NETs clearance dysfunction can lead to a variety of autoimmune diseases, such as systemic lupus erythematous, rheumatoid arthritis, Felty's syndrome and small vessel vasculitis (Kessenbrock et al., 2009; Darrah and Andrade, 2012). Also, NETs plays an important role in the process of hemostasis and thrombosis, which is closely related to the formation of venous thrombosis (Manfredi et al., 2010). The plasma markers levels of NETs are related to the severity of thrombus diseases, such as thrombotic micro-angiopathy (Fuchs et al., 2012) and coronary atherosclerosis (Borissoff et al., 2013).

\section{NETs and tumor cell proliferation, distant metastasis and angiogenesis}

NETs are highly expressed in many malignant tumors. There are NETs structures in lung cancer tissues and human osteosarcoma tissues (Berger-Achituv et al., 2013; Demers and Wagner, 2013). In the murine model of breast cancer, non-small cell lung cancer and chronic myeloid leukemia, the neutrophils are more prone to release NETs structure (Demers et al., 2012). Many of the characteristics of NETs make it possible to regulate the biological process of malignant diseases.

Studies show that NETs can directly promote tumor cell proliferation, distant metastasis and angiogenesis. As mentioned above, NETs are composed of chromatin and antibacterial proteins, peptides, including MMP-9, CG, NE, etc. The high concentrations of antimicrobial peptides in the local microenvironment of NETs are indispensable for elimination of pathogen. Similar to such a process, the role of multiple proteins in NETs microenvironment is worthy of further study. The study confirmed that MMP-9 was involved in tumor growth and metastasis, including inhibiting apoptosis and promoting angiogenesis (Bergers et al., 2000; Masson et al., 2005). $\mathrm{CG}$ could promote angiogenesis (Wilson et al., 2010) and NE had a direct effect on tumor cell proliferation and distant metastasis (Houghton et al., 2010). Therefore, it can be speculated that NETs can capture circulating tumor cells. Tumor cells are exposed to the microenvironment with high concentration of multiple proteins, which can promote tumor cells proliferation, distant metastasis and angiogenesis, and inhibit cell apoptosis.

Similar to the process of capturing the pathogenic microorganisms, NETs can anchor the circulating tumor cells, and help them to evade the immune system. At the same time NETs and the degradation products, which can inhibit immune cell function (Huh et al., 2010; CoolsLartigue et al., 2013). Animal experiments confirmed that the induction of NETs could promote formation of metastatic nodules in the lungs and liver; and the use of the DNase enzyme cutting the DNA backbone of NETs, or NE inhibitors which inhibit the formation of NETs, can significantly reduce the formation of distant organ metastasis nodule (Cools-Lartigue et al., 2013).

At the same time, NETs can also through physical interaction, anchor circulating tumor cells to endothelial cells, which is conducive for tumor cells and endothelial cell adhesion and diapedesis; and interference interactions between neutrophils and endothelial cells, can effectively reduce tumor cell transfer across endothelial exudation and distant organs metastasis (Cools-Lartigue et al., 2013; Cools-Lartigue et al., 2014). It has been confirmed that mice injected with neutrophils can lead to increased adhesion ability of tumor cells to the liver capillary sinus and lung capillary by nearly 5 times. However, in neutrophil deficient mice, the adhesion of tumor cell to endothelial cell was significantly inhibited (Spicer et al., 2012).

Therefore, NETs provides microenvironment to promote tumor cell proliferation, distant metastasis and angiogenesis. By anchoring the circulating tumor cells, NETs help tumor cells escape from the immune attack; also, NETs helps to adhere to endothelial cells, which is conducive to tumor cell exudation.

\section{NETs and tumor associated thrombosis}

The incidence of deep venous thrombosis in patients with malignant tumor is about $10-30 \%$. Tumor associated thrombosis seriously affects the life quality and survival of cancer patients (Sorensen et al., 2000; Goldhaber and Tapson, 2004). Thus, intervention of NETs has an important clinical meaning. NETs and platelet interactions are involved in the formation of thrombosis and tumor related thrombosis, which provides a new target for clinical evaluation and treatment of thrombosis (Sorensen et al., 2000; Goldhaber and Tapson, 2004).

NETs were found in both plasma and thrombus, suggesting that it can be used as a part of the thrombus structure (Longstaff et al., 2013). Similarly, the NETs structure was found in human venous thrombosis (Nakazawa et al., 2012). Even more important, PAD4 gene deficient mice confirmed that NETs was not only a part of venous thrombosis, but also played an important role in the formation and maintenance of the thrombus. PAD4 gene deficient mice could not form thrombosis; on the other hand, infused with wild type neutrophils, PAD4 deficient could also form venous thrombosis (Martinod et al., 2013). 
Studies show that NETs can participate in a variety of pathways leading to thrombosis (Demers and Wagner, 2013). Similar to Willebrand-von factor and fibrin, as a macromolecular complex, NETs can provide a mesh stent structure for platelet adhesion and aggregation (Nakazawa et al., 2012; Longstaff et al., 2013). Moreover, a variety of components in NETs, including the nuclear DNA, histone and protease, have the coagulant activity. In vitro experiments demonstrated that extracellular DNA could enhance the activity of proteinase (Kannemeier et al., 2007). Histones are toxic to endothelial cells, involved in the formation of diffuse micro-thrombus and deposition of fibrin and platelet (Xu et al., 2009). By inhibiting the plasma anticoagulant activity, histones promote platelet aggregation (Ammollo et al., 2011). By inhibiting the tissue factor pathway, NE can enhance thrombin activity and promote the fibrin deposition (Massberg et al., 2010). In patients with sepsis, platelets can be activated by TLR4, and then combined with the neutrophils to produce NETs, resulting in tissue damage and abnormal coagulation function (Clark et al., 2007). Thus, the NETs can provide a scaffold for platelet adhesion and aggregation, and a variety of components in NETs can promote the coagulation activity.

NETs were closely related to tumor-associated thrombosis (Demers et al., 2012; Demers and Wagner, 2014).In many different kinds of malignant tumors, there are plasma free DNA and NETs, which are closely related to the formation of spontaneous thrombosis (Demers et al., 2012; Demers and Wagner, 2014). The injection of endotoxin in the tumor bearing mice can induce the formation of NETs, which can induce the thrombus and the blood coagulation function. Neutrophil analysis in tumor bearing mice peripheral, confirmed the histone citrullination level is higher, and compared with the normal neutrophils, neutrophil is prone to the formation of NETs (Demers et al., 2012); by DNase digestion pretreatment can prevent the occurrence of the endotoxin injection caused NETs and pulmonary thrombosis in tumor bearing mice (Demers et al., 2012).

Neutrophils release NETs with the presence of highly expressed PAD4. PAD4 expression level and citrullinated histone levels can reflect the level of NETs in vivo (Li et al., 2010). Tumor associated thrombosis are usually associated with the increased number of highly citrullinated neutrophil counts in the circulation (Demers et al., 2012; Demers and Wagner, 2014). And tumor associated thrombotic micro-vascular disease patients also showed that high level of citrullinated histone in plasma, while not in healthy control group (Demers et al., 2012; Fuchs et al., 2012). Therefore, the level of highly citrullinated neutrophil count, and level of citrullinated histone in plasma, can be used as biomarkers to evaluate the formation of spontaneous thrombosis in tumor patients. However, the use of NETs to predict the risk of tumor associated thrombosis, its sensitivity and specificity, still need to be further studied in large clinical samples.

\section{NETs as a potential therapeutic target for cancer}

NETs have been involved in tumor cell adhesion,
Progression of NETs Correlating with Tumor-related Diseases

proliferation, distant metastasis and tumor associated thrombosis. Thus, it can be speculated that the degradation or inhibition of NETs has potential therapeutic effect in tumor related diseases.

The research shows that the formation and degradation of NETs is a complicated process regulated by many factors (Brinkmann et al., 2004). PAD4 has been found over-expression in human malignant tumors (Chang and Han, 2006; Chang et al., 2009). It is worth noting that PAD4 can induce NETs structure in cancer cells (Leshner et al., 2012), while PAD4 inhibitors can promote the expression of P53 gene and inhibit tumor cell proliferation, induce apoptosis, and reduce the incidence of pulmonary thrombosis ( $\mathrm{Li}$ et al., 2008; Li et al., 2010). However, whether PAD4 inhibitor can be used for cancer treatment through the formation of NETs or not, remains to be confirmed.

In addition, in vivo studies confirmed that systemic inflammatory response could promote tumor cells distant organ metastasis. NE inhibitors or DNase can significantly inhibit the circular NETs formation and reduce adhesion of tumor cells to capillary in the liver sinus and lung capillary endothelial cell (Cools-Lartigue et al., 2013). DNase has been approved for the treatment of cystic fibrosis and empyema (Makino et al., 2011). In view of this, DNase can be used to treat tumor related thrombosis, and even to inhibit tumor cell proliferation and metastasis. However, the clinical use of DNase still needs to be further studied.

Similarly, could NE and PAD4 inhibitors inhibit the formation of NETs in vivo? Whether NE and PAD4 inhibitors can be used in tumor associated thrombosis, or even the tumor itself, still need to be further studied. In addition, whether the NETs inhibitor has a synergistic effect with chemotherapy, radiotherapy, targeted therapy and immunotherapy, still largely unknown.

\section{Conclusion and Prospect}

NET opens up a new research field for the study of tumor biological behavior and provides a novel explanation for the interaction among tumor and inflammation and thrombosis. Activated neutrophils release NETs, which is involved in tumor progression through multiple pathways, and the use of DNase or NE or PAD4 inhibitors, may be a potential therapeutic target by blocking the formation of circular NETs. However, whether the NETs inhibitor has a therapeutic effect in tumor related diseases is still to be confirmed by further studies.

\section{References}

Ammollo CT, Semeraro F, Xu J, et al (2011). Extracellular histones increase plasma thrombin generation by impairing thrombomodulin-dependent protein C activation. J Thromb Haemost, 9, 1795-803.

Amulic B, Cazalet C, Hayes GL, et al (2012). Neutrophil function: from mechanisms to disease. Annu Rev Immunol, 30, 459-89.

Berger-Achituv S, Brinkmann V, Abed UA, et al (2013). A proposed role for neutrophil extracellular traps in cancer immunoediting. Front Immunol, 4, 48.

Bergers G, Brekken R, McMahon G, et al (2000). Matrix 
metalloproteinase- 9 triggers the angiogenic switch during carcinogenesis. Nat Cell Biol, 2, 737-744.

Borissoff JI, Joosen IA, Versteylen MO, et al (2013). Elevated levels of circulating DNA and chromatin are independently associated with severe coronary atherosclerosis and a prothrombotic state. Arterioscler Thromb Vasc Biol, 33, 2032-40.

Brandau S, Dumitru CA, Lang S (2013). Protumor and antitumor functions of neutrophil granulocytes. Semin Immunopathol, 35, 163-76.

Brinkmann V, Reichard U, Goosmann C, et al (2004). Neutrophil extracellular traps kill bacteria. Science, 303, 1532-5.

Chang X, Han J (2006). Expression of peptidylarginine deiminase type 4 (PAD4) in various tumors. Mol Carcinog, 45, 183-96.

Chang X, Han J, Pang L, et al (2009). Increased PADI4 expression in blood and tissues of patients with malignant tumors. Bmc Cancer, 9, 40.

Clark SR, Ma AC, Tavener SA, et al (2007). Platelet TLR4 activates neutrophil extracellular traps to ensnare bacteria in septic blood. Nat Med, 13, 463-9.

Cools-Lartigue J, Spicer J, McDonald B, et al (2013). Neutrophil extracellular traps sequester circulating tumor cells and promote metastasis. J Clin Invest.

Cools-Lartigue J, Spicer J, Najmeh S, et al (2014). Neutrophil extracellular traps in cancer progression. Cell Mol Life Sci, 71, 4179-94.

Darrah E, Andrade F (2012). NETs: the missing link between cell death and systemic autoimmune diseases? Front Immunol, 3, 428 .

Demers M, Krause DS, Schatzberg D, et al (2012). Cancers predispose neutrophils to release extracellular DNA traps that contribute to cancer-associated thrombosis. Proc Natl Acad Sci U S A, 109, 13076-81.

Demers M, Wagner DD (2013). Neutrophil extracellular traps: A new link to cancer-associated thrombosis and potential implications for tumor progression. Oncoimmunol, 2, 22946.

Demers M, Wagner DD (2014). NETosis: a new factor in tumor progression and cancer-associated thrombosis. Semin Thromb Hemost, 40, 277-83.

Fuchs TA, Abed U, Goosmann C, et al (2007). Novel cell death program leads to neutrophil extracellular traps. J Cell Biol, 176, 231-41.

Fuchs TA, Kremer HJ, Schatzberg D, et al (2012). Circulating DNA and myeloperoxidase indicate disease activity in patients with thrombotic microangiopathies. Blood, 120, 1157-64.

Goldhaber SZ, Tapson VF (2004). A prospective registry of 5,451 patients with ultrasound-confirmed deep vein thrombosis. Am J Cardiol, 93, 259-62.

Gregory AD, Houghton AM (2011). Tumor-associated neutrophils: new targets for cancer therapy. Cancer Res, 71, 2411-6.

Houghton AM, Rzymkiewicz DM, Ji H, et al (2010). Neutrophil elastase-mediated degradation of IRS-1 accelerates lung tumor growth. Nat Med, 16, 219-23.

Huh SJ, Liang S, Sharma A, et al (2010). Transiently entrapped circulating tumor cells interact with neutrophils to facilitate lung metastasis development. Cancer Res, 70, 6071-82.

Kannemeier C, Shibamiya A, Nakazawa F, et al (2007). Extracellular RNA constitutes a natural procoagulant cofactor in blood coagulation. Proc Natl Acad Sci U S A, 104, 6388-93.

Kessenbrock K, Krumbholz M, Schonermarck U, et al (2009). Netting neutrophils in autoimmune small-vessel vasculitis. Nat Med, 15, 623-5.

Leshner M, Wang S, Lewis C, et al (2012). PAD4 mediated histone hypercitrullination induces heterochromatin decondensation and chromatin unfolding to form neutrophil extracellular trap-like structures. Front Immunol, 3, 307.

Li $P$, Li M, Lindberg MR, et al (2010). PAD4 is essential for antibacterial innate immunity mediated by neutrophil extracellular traps. J Exp Med, 207, 1853-62.

Li $P$, Wang D, Yao H, et al (2010). Coordination of PAD4 and HDAC2 in the regulation of p53-target gene expression. Oncogene, 29, 3153-62.

Li $P$, Yao H, Zhang Z, et al (2008). Regulation of p53 target gene expression by peptidylarginine deiminase 4 . Mol Cell Biol, 28, 4745-58.

Longstaff C, Varju I, Sotonyi $P$, et al (2013). Mechanical stability and fibrinolytic resistance of clots containing fibrin, DNA, and histones. J Biol Chem, 288, 6946-56.

Makino H, Kunisaki C, Kosaka T, et al (2011). Perioperative use of a neutrophil elastase inhibitor in video-assisted thoracoscopic oesophagectomy for cancer. Br J Surg, 98, 975-82.

Manfredi AA, Rovere-Querini $P$, Maugeri N (2010). Dangerous connections: neutrophils and the phagocytic clearance of activated platelets. Curr Opin Hematol, 17, 3-8.

Mantovani A (2009). The yin-yang of tumor-associated neutrophils. Cancer Cell, 16, 173-4.

Martinod K, Demers M, Fuchs TA, et al (2013). Neutrophil histone modification by peptidylarginine deiminase 4 is critical for deep vein thrombosis in mice. Proc Natl Acad Sci U S A, 110, 8674-9.

Massberg S, Grahl L, von Bruehl ML, et al (2010). Reciprocal coupling of coagulation and innate immunity via neutrophil serine proteases. Nat Med, 16, 887-96.

Masson V, de la Ballina LR, Munaut C, et al (2005). Contribution of host MMP-2 and MMP-9 to promote tumor vascularization and invasion of malignant keratinocytes. Faseb J, 19, 234-6.

Metzler KD, Fuchs TA, Nauseef WM, et al (2011). Myeloperoxidase is required for neutrophil extracellular trap formation: implications for innate immunity. Blood, 117, 953-9.

Nakazawa D, Tomaru U, Yamamoto C, et al (2012). Abundant neutrophil extracellular traps in thrombus of patient with microscopic polyangiitis. Front Immunol, 3, 333.

Palmer LJ, Cooper PR, Ling MR, et al (2012). Hypochlorous acid regulates neutrophil extracellular trap release in humans. Clin Exp Immunol, 167, 261-8.

Papayannopoulos V, Metzler KD, Hakkim A, et al (2010). Neutrophil elastase and myeloperoxidase regulate the formation of neutrophil extracellular traps. J Cell Biol, 191, 677-91.

Sorensen HT, Mellemkjaer L, Olsen JH, et al (2000). Prognosis of cancers associated with venous thromboembolism. NEngl $J$ Med, 343, 1846-50.

Spicer JD, McDonald B, Cools-Lartigue JJ, et al (2012). Neutrophils promote liver metastasis via Mac-1-mediated interactions with circulating tumor cells. Cancer Res, 72, 3919-27.

Wang Y,Li M, Stadler S, et al (2009). Histone hypercitrullination mediates chromatin decondensation and neutrophil extracellular trap formation. J Cell Biol, 184, 205-13.

Wilson TJ, Nannuru KC, Futakuchi M, et al (2010). Cathepsin G-mediated enhanced TGF-beta signaling promotes angiogenesis via upregulation of VEGF and MCP-1. Cancer Lett, 288, 162-9.

Xu J, Zhang X, Pelayo R, et al (2009). Extracellular histones are major mediators of death in sepsis. Nat Med, 15, 1318-21. 